\title{
Shock parameter calculations at weak interplanetary shock waves
}

\author{
J. M. Gloag and A. Balogh \\ Imperial College Space and Atmospheric Group Department of Physics, Prince Consort Road, London SW7 2BW, UK
}

Received: 31 March 2004 - Revised: 18 October 2004 - Accepted: 19 November 2004 - Published: 28 February 2005

\begin{abstract}
A large set of interplanetary shock waves observed using the Ulysses spacecraft is analysed in order to determine their local parameters. For the first time a detailed analysis is extended to the thermodynamic properties of a large number of events. The intention is to relate the shock parameters to the requirements set by MHD shock theory. A uniform approach is adopted in the selection of up and downstream regions for this analysis and applied to all the shock waves. Initially, the general case of a 3 component adiabatic plasma is considered. However, the calculation of magnetosonic and Alfvénic Mach numbers and the ratio of downstream to upstream entropy produce some unexpected results. In some cases there is no clear increase in entropy across the shock and also the magnetosonic Mach number can be less than 1 . It is found that a more discerning use of data along with an empirical value for the polytropic index can raise the distribution of downstream to upstream entropy ratios to a more acceptable level. However, it is also realised that many of these shocks are at the very weakest end of the spectrum and associated phenomena may also contribute to the explanation of these results.
\end{abstract}

Key words. Interplanetary physics (Interplanetary shocks, discontinuities)

\section{Introduction}

Around the time of the first identification of a collisionless shock wave in the heliosphere by Sonett et al. (1964) the actual existence of shock waves supporting dissipation with no collisions was in question. The many observations of shock waves by various spacecraft, in the following years confirmed the presence of the shocks in space and revealed a whole spectrum of different shock structures. These discontinuous structures varied from the strong planetary bow shocks to the weak and sometimes debatable interplanetary shock waves. The theoretical foundation for these magnetohydrodynamic shock waves was provided by de Hoffmann and Teller (1950), who extended the existing fluid theory to accommodate a magnetised plasma. The MHD shock wave description sets out clear conditions that must be satisfied

Correspondence to: J. M. Gloag

(jonathan.gloag@imperial.ac.uk) for a particular event seen in spacecraft data to be described as a shock wave. This theoretical shock structure consists of an increase in magnetic field magnitude and deflection of the magnetic field away from the shock normal, in the forward direction, for the much more commonly observed fast mode shock. In the case of slow mode shocks, passing from upstream to downstream, the magnetic field magnitude decreases and the field bends towards the shock normal direction. In both cases, in the shock stationary normal incidence frame of reference, the plasma velocity falls from above to below the relevant linear wave speed and also the plasma entropy increases. This entropy increase is caused by an irreversible compression (increase in plasma density) and heating of the plasma. These conditions can be expressed in terms of shock parameters which require the use of both magnetic and plasma data measured in situ by spacecraft experiments. In an ideal MHD case the plasma regions on either side of the shock wave are well defined, equilibrium states. This is generally not the case when shock waves are observed in their natural environment.

Strong shock waves, such as the Earth's bow shock with a typical magnetosonic Mach number of 8 , are observed to have clear temperature and density structure, although the magnetic field is subject to more perturbing fluctuations. Weak interplanetary shocks with magnetosonic Mach numbers in the range of 1-4 often have less clear plasma signatures and again, the magnetic field can contain large disturbances. To gain a proper understanding of these weaker events both shock induced fluctuations, caused by dissipation effects leaking from the thin dissipation layer, and constantly arising solar wind fluctuations must be taken into consideration. The selection of up and downstream regions for use in the calculation of shock parameters is a long standing problem. This problem has been confronted in connection with the calculation of $\theta_{B n}$ on several occasions where it is the higher time resolution magnetic field data that has allowed for the flexibility to carry out the investigations, Chao and Hsieh, 1984; Hsieh and Richter, 1986; Liu et al., 1996; Gonzáles-Esparza and Balogh, 2001. Another problem is connected with the determination of plasma parameters and specifically temperatures near shock waves. This can be particularly difficult in the outer heliosphere in cold plasma conditions where care must be taken to use appropriate parameter values. 

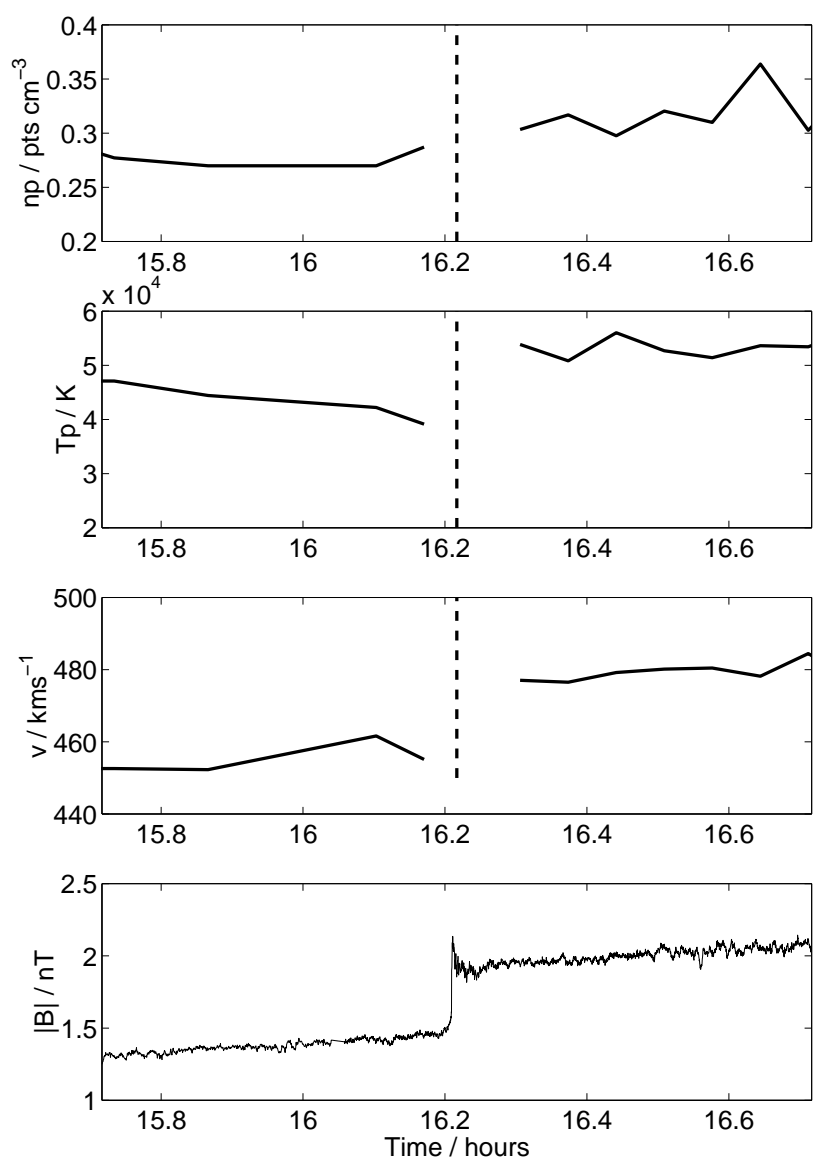

Fig. 1. Example low $r_{B}$ shock occurring on day 128, 1998 showing proton density (top panel), proton temperature (2nd panel), proton bulk velocity (3rd panel) and magnetic field magnitude (bottom panel).

In this study over 100 interplanetary shock waves are analysed, all of which have been observed by the Ulysses spacecraft between 1996 and 1999. The analysis consists of applying a set of up and downstream regions to all the shocks methodically in order to calculate their local shock parameters. The size of the plasma regions have been chosen as a compromise so as not to be dominated by fluctuations produced by the shock but also to minimise the inclusion of solar wind fluctuations. These shock parameters are then compared to the conditions set by the theory. It is found that the agreement of some of the shock parameters with the theory depends specifically on the choice of data used in the analysis and on assumptions made about the thermodynamic properties of the solar wind plasma. The choice of data that can be used for the analysis is related to the difficulties of calculating the plasma temperature in a collisionless plasma. The discrepancy between the analysis and theory is partly an expression of the fact that the events used here are weak interplanetary shocks and in some cases at the extreme weak end of the spectrum.

\section{Data analysis}

The shock waves used in this work are present on the Ulysses shock list (private communication, R. J. Forsyth and J. T. Gosling, http://www.imperial.ac.uk/research/spat/ research/ulysses/shocklist.txt) between August 1996 and the end of 1999. During this period of time Ulysses passed from above to below the ecliptic plane at the furthest point from the Sun on its polar orbit. Since Ulysses swung by Jupiter in 1992 it has been in an orbit which is inclined $79.4^{\circ}$ to the ecliptic plane and has a period of 6.2 years. For shocks to appear on the shock list a discontinuous change in the magnetic field magnitude must be observed to occur over a few seconds of data. There must also be a corresponding heating, compression and change in bulk velocity of the plasma. The changes in the plasma parameters cannot be observed to occur over the same time scale as the magnetic parameters due to the lower time resolution of the plasma measurements. Plasma parameters are generated every 4 or $8 \mathrm{~min}$ (Bame et al., 1992) compared to either 1 or $2 \mathrm{sec}$ (Balogh et al., 1992) for the magnetometer data. The difficulties encountered due to these data time resolutions have been faced in a previous study of interplanetary shocks observed by Ulysses between 1990 and 1993 (Balogh et al., 1995). The low $r_{B}$ values and low Mach numbers, some of which were calculated to be less than 1, already demonstrate in this previous work that these interplanetary shocks under consideration are weak structures.

The size of the up and downstream regions to be used in the calculation of the shock parameters for this study is influenced to some extent by the resolution of the plasma data. For magnetic parameters, $10 \mathrm{~min}$ of data are used on either side of the shocks and 2 plasma data points are used regardless of the time resolution, in order to define a representative plasma state. The magnetic parameters calculated in this work are $\theta_{B n}$, the angle between the shock normal vector and the upstream magnetic field, $\theta_{\mathrm{Bdn}}$, the angle between the shock normal and the downstream magnetic field, and $r_{B}$ which is the ratio of downstream to upstream magnetic field magnitude. The shock normal and therefore $\theta_{B n}$ and $\theta_{\mathrm{Bdn}}$ are calculated using the magnetic coplanarity theorem (Colburn and Sonett, 1966). Following on from a statistical approach to calculate $\theta_{B n}$ (González-Esparza, 1995), it is found that for the purposes of this study, the use of averaged 10-min up and downstream regions is adequate. Figures 1 and 2 are example shocks with low and high $r_{B}$ values, respectively. These two figures demonstrate the difference in time resolution between the magnetic and plasma data. It is also apparent that, although in both the weak and strong case the magnetic shock signature is clear, the plasma transition is much less distinct in the low $r_{B}$ case. Parameter calculations that require the use of plasma data are Alfvén and magnetosonic Mach numbers and the ratio of downstream to upstream entropy. The Mach numbers are the ratio of plasma velocity to a particular linear wave speed in a stationary reference frame normal to the shock front. The shock velocities needed to determine these Mach numbers are calculated using the 

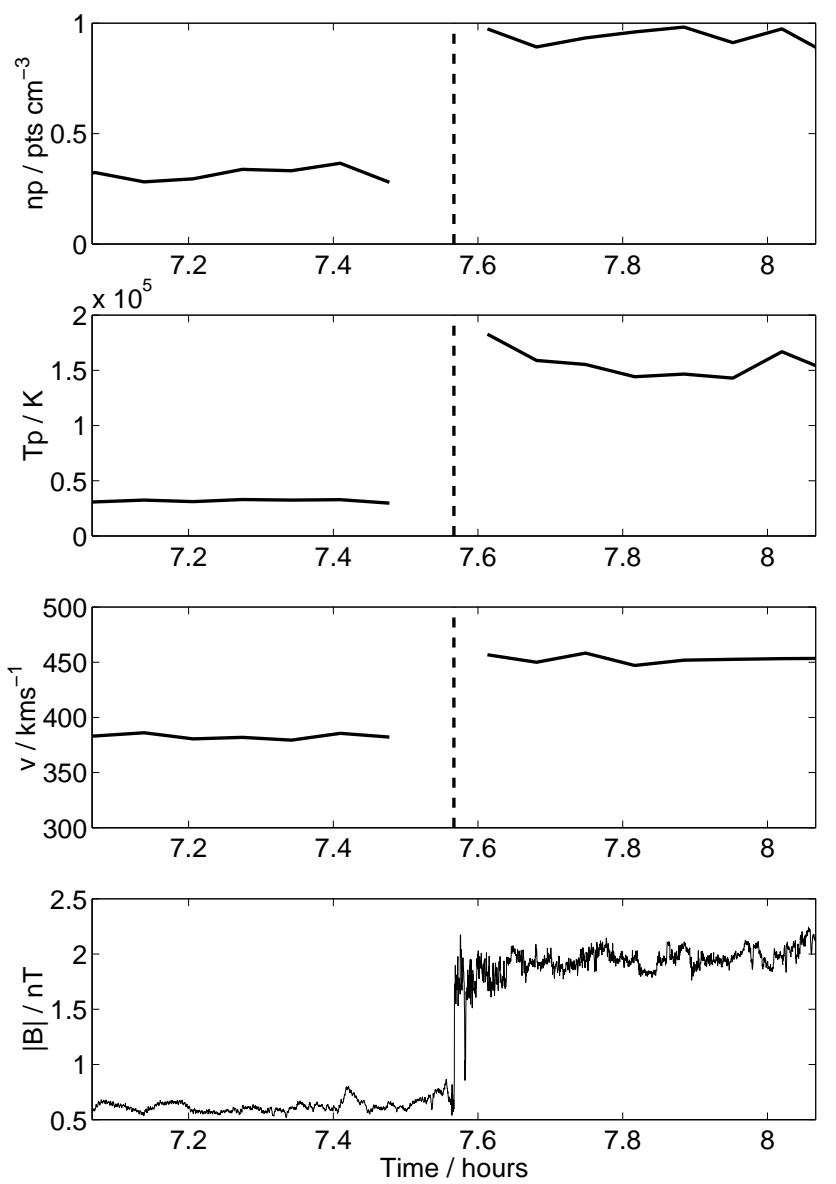

Fig. 2. Example high $r_{B}$ shock occurring on day 146, 1999 showing proton density (top panel), proton temperature (2nd panel), proton bulk velocity (3rd panel) and magnetic field magnitude (bottom panel).

Smith and Burton method (Smith and Burton, 1988). Equation (1) (below) is used to check for entropy increases across the shock waves. The right-hand side divided by the lefthand side of Eq. (1) gives the downstream to upstream ratio of the entropy argument. The entropy for a particular plasma state is actually proportional to $\ln \left(\frac{T^{\frac{1}{(\gamma-1)}}}{n}\right)$ but in this work the argument of this expression is used. Equation (1) is written for a three-component plasma consisting of protons, subscript $\mathrm{p}$, alpha particles, $\alpha$ and electrons, e. $T$ and $n$ are the temperature and number density for each of the particle species and $\gamma$ is the ratio of specific heat capacities. The subscripts $\mathrm{u}$ and $\mathrm{d}$ refer to the up and downstream regions, respectively.

$\frac{n_{p d} T_{p d}+n_{e d} T_{e d}+n_{\alpha d} T_{\alpha d}}{n_{p u} T_{p u}+n_{e u} T_{e u}+n_{\alpha u} T_{\alpha u}}>\left(\frac{n_{p d}+n_{e d}+n_{\alpha d}}{n_{p u}+n_{e u}+n_{\alpha u}}\right)^{\gamma}$

Entropy calculations at shock waves have received only a small amount of attention in the past. In fact, the assumption that entropy increases at shocks measured in this way has featured as a test in the identification of possible slow
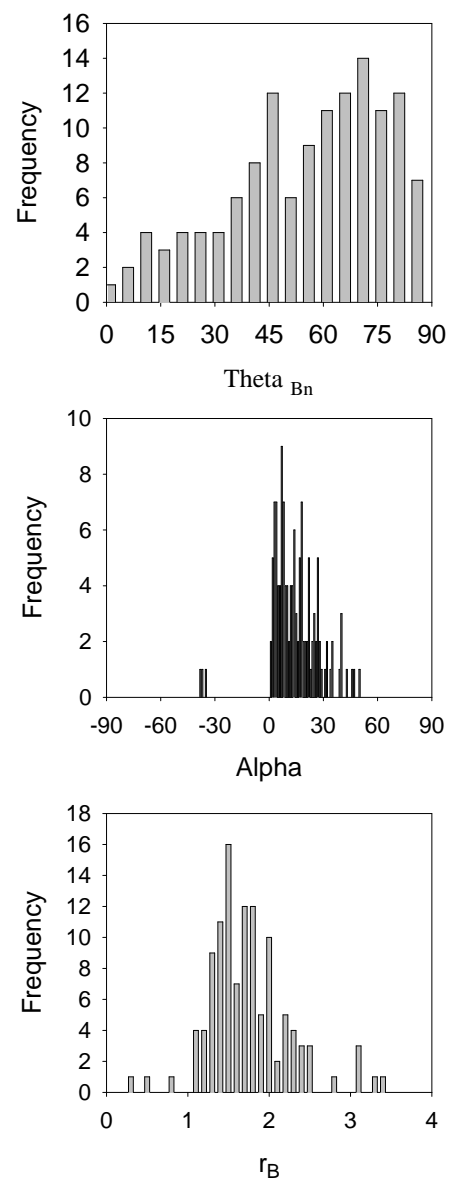

Fig. 3. Magnetic parameters for all shock waves considered in this survey using 10-min averaged data for up and downstream regions and using the magnetic coplanarity method. Top panel: Histogram of $\theta_{B n}$ values. Middle panel: Histogram of $\alpha$ the magnetic deflections. Bottom panel: Histogram of magnetic compressions.

shocks. For instance, Richter et al. (1985) used a similar expression to Eq. (1) for precisely this use. Previously to this Chao (1973) used a two-component plasma expression with the added simplifying condition of quasi-neutrality in an investigation of nonlinear wave steepening in the solar wind.

The calculation of the previously described shock parameters has shown that in every case the magnetic parameters agree with the predictions set out by the theory. More precisely this means that magnetic field deflections are in the correct direction and $r_{B}$ values are in the expected ranges for fast and slow mode shocks, as set out in the Introduction. Figure 3 shows histograms of $\theta_{B n}$ values, deflections, $\alpha$, and magnetic compressions for all the shock waves under consideration. The magnetic deflections are calculated as the difference between $\theta_{\mathrm{Bdn}}$ and $\theta_{B n}$. The histogram of $\theta_{B n}$ values in the top panel shows that there are more quasi-perpendicular shock waves than quasi-parallel shock waves which would be expected at the kind of distances from the Sun where Ulysses is found. The middle panel shows that all but three events have positive deflections. This result when taken with 

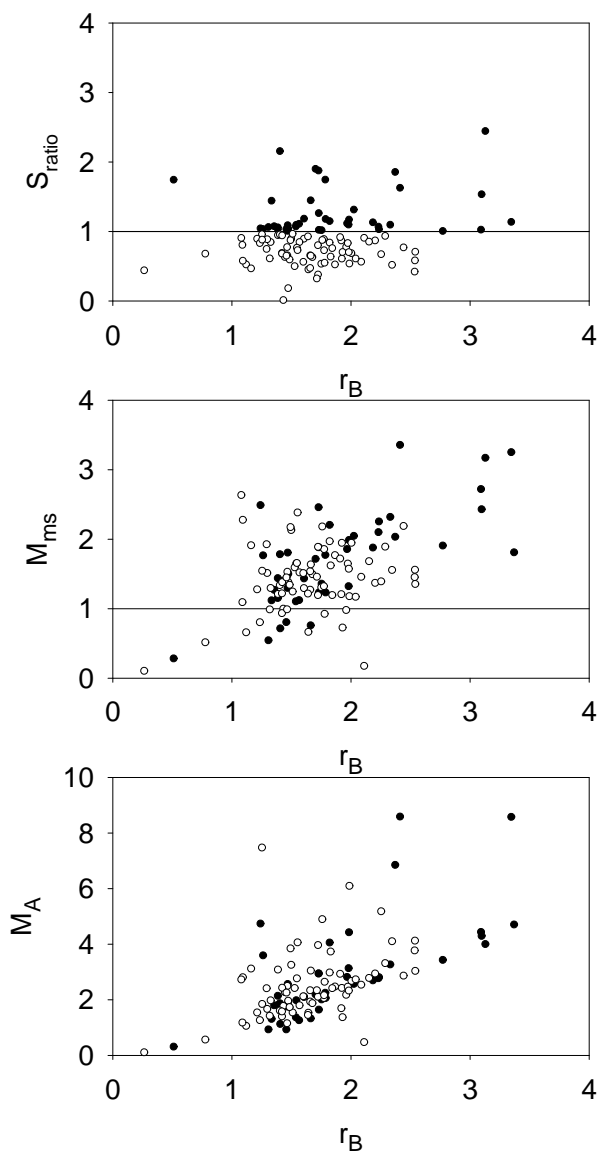

Fig. 4. These results are obtained using 2-plasma point up and downstream data periods for a 3-component plasma and $\gamma=\frac{5}{3}$. Top panel shows the entropy ratio against $r_{B}$, middle panel the magnetosonic Mach number against $r_{B}$, and bottom panel the Alfvén Mach number against $r_{B}$. In all cases filled circles have entropy increases and open circles have entropy decreases.

the histogram of magnetic compression ratios in the bottom panel of Fig. 3 shows that all but three events are fast mode shock waves. The three exceptions which each have negative deflections and $r_{B}$ values less than 1 are three slow shock candidates. Although the calculated magnetic parameters agree with the theory, it is found that some shock waves appear not to follow the expected results in terms of Mach numbers and entropy ratios. Figure 4 demonstrates these unexpected results where entropy calculations have been made using data for a three-component plasma and assuming $\gamma=\frac{5}{3}$. The proton temperatures used in this case are the average of the two temperatures present in the SWOOPS data which are determined in different ways and in general bracket the true proton temperature. The method that tends to overestimate the proton temperature is the integral over all energy channels and angular bins of the three-dimensional velocity distribution space. The alternative method uses the radial component of the temperature tensor for which the calculation involves summing over angular bins at fixed energy and this tends to underestimate the proton temperature. The
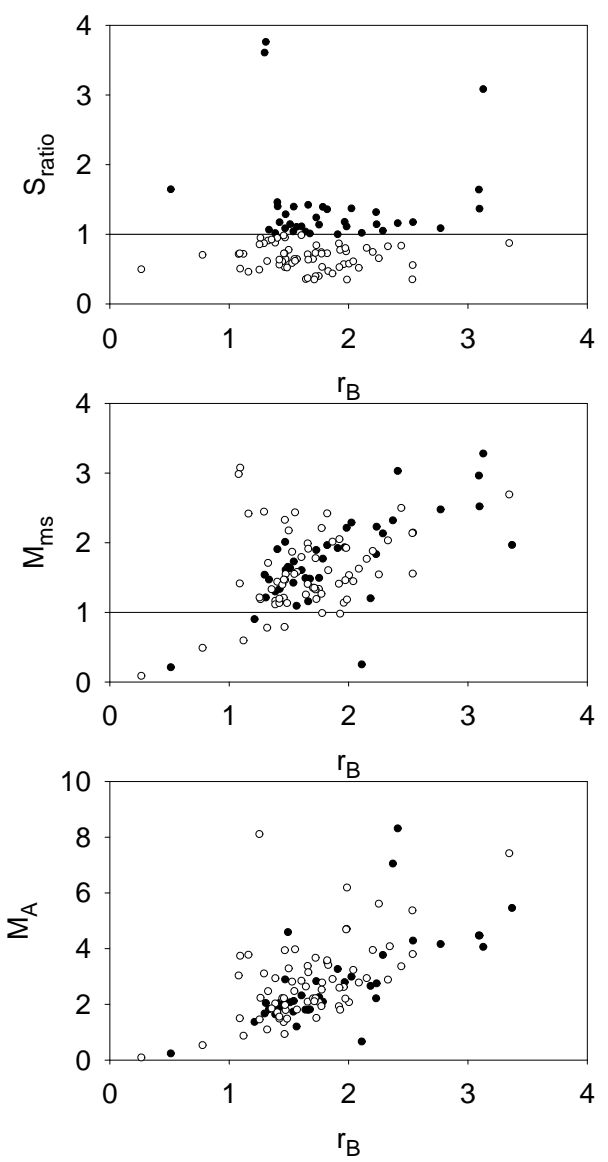

Fig. 5. These results are obtained using 1-h up and downstream data periods for a 3 -component plasma and $\gamma=\frac{5}{3}$. Top panel shows the entropy ratio against $r_{B}$, middle panel the magnetosonic Mach number against $r_{B}$, and bottom panel the Alfvén Mach number against $r_{B}$. Filled circles have entropy increases and open circles have entropy decreases.

temperature of the $\alpha$ particles is not derived directly from the distribution functions and does not appear in the plasma data. The empirical relation $T_{\alpha}=3.9 T_{p}$ is used here for the $\alpha$ particle temperature and this was determined by Liu et al. (1995). The most striking result from Fig. 4 is that over 50\% of the shock waves appear to have entropy decreases across their boundaries which makes this result difficult to accept.

Given the fact that the entropy is expected to increase from up to downstream at all shock waves, the next step in the investigation is to try and find the source of the apparent entropy decrease problem. It is possible that the 10-min up and downstream regions which have been found to be suitable to analyse interplanetary shock waves in terms of magnetic parameters are too short for the plasma parameter analysis. If this is the case then the entropy increase at these shock waves may only be visible over longer periods of time. To investigate this possibility entropy ratios have been calculated using 1-h up and downstream regions. These calculations have again been done using the average of the two proton temperature estimations, assuming an up and downstream adiabatic 
plasma with $\gamma=\frac{5}{3}$ and for a three component plasma. The resulting entropy ratios are shown in Fig. 5 and it can be seen from this that the longer up and downstream periods do not resolve this entropy problem.

It has been found by looking at electron temperatures across the shock waves that in many cases the electrons do not behave as predicted in that they do not heat adiabatically. Considering purely the electron heating expected from adiabatic compression, there are 75 events which do not attain a high enough downstream temperature to be within $10 \%$ of the expected value. The electron temperature expected to be attained from conservation of the magnetic moment is not achieved to within $10 \%$ in 103 cases. In fact, in 39 of the shocks which are calculated to have entropy decreases, the electrons appear to cool. It is important to note that the solar wind conditions encountered by Ulysses during the period being considered in this paper may not always lead to completely reliable electron parameters (private communication, B. Goldstein, JPL, 13 March 2001). Another factor which could affect these results is the choice of $\gamma$. Totten and Freeman (1995) have shown that a value closer to 1.5 may be more appropriate for the solar wind. This value for $\gamma$ was calculated empirically using Helios 1 data. Finally, due to the higher energy resolution of the instrument from which data is used to determine proton temperatures, as opposed to the angular resolution, the radial temperatures may be closer to the true proton temperature (private communication, J. Gosling, LANL, 21 May 2001). The radial temperatures correspond to the lower of the two proton temperatures previously mentioned which were used together to calculate the average temperature to produce the results in Fig. 4. We have recalculated the shock parameters using only proton data, $\gamma=1.5$ and radial proton temperatures. These results are shown in Fig. 6 and show that in this case most of the shocks now have entropy increases with only 14 shocks just below the boundary.

It has been shown that by careful selection of data the number of shock waves failing the entropy test for a shock wave can be minimised. Although using the empirical value of $\gamma=1.5$ increases the number of shock waves which are calculated to have entropy increases across their boundaries, it is not clear that this is the correct thing to do from a physical point of view. The difference between the empirical value of $\gamma$ and the adiabatic value can be explained by a heating effect which has a measurable presence in the solar wind. As the solar wind expands through the heliosphere it cools as well. However, the cooling is less than that expected from adiabatic expansion due to an electron heat flux which is explained by a hot population of electrons streaming along the heliospheric magnetic field lines directly from the corona (Gosling et al., 1973; Totten and Freeman, 1995). These processes explain the properties of the solar wind plasma on a large heliospheric scale. This understanding allows us to realise that the empirical value of $\gamma$ is not appropriate in the case of shock wave studies and that a value is needed that reflects properties of the plasma local to a shock wave. From a theoretical point of view larger values of $\gamma$ are thought to
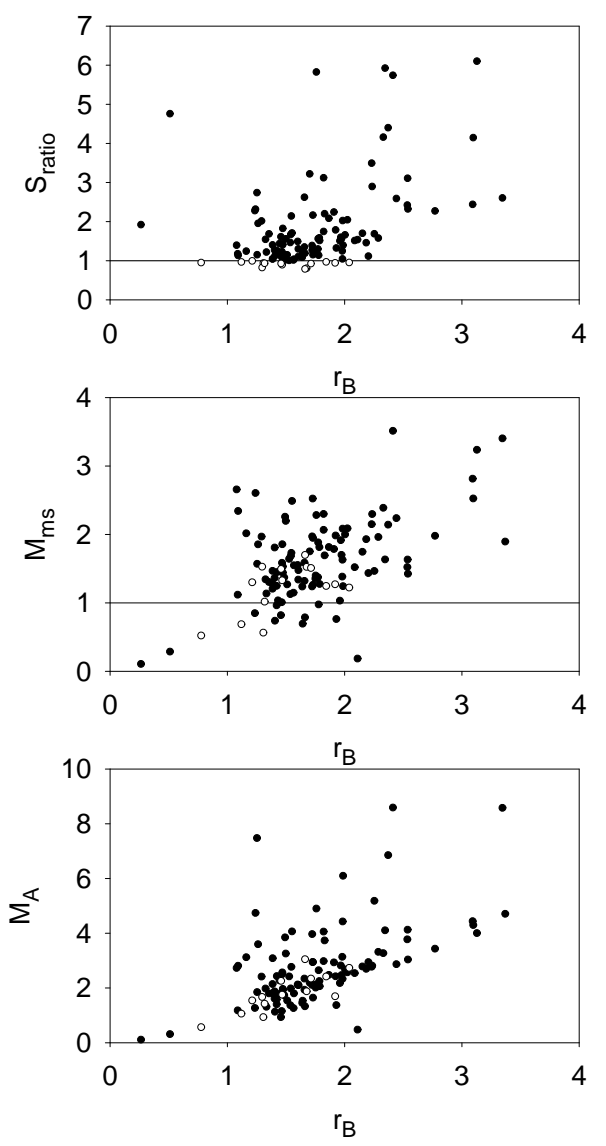

Fig. 6. These results are obtained using 2-plasma point up and downstream data periods, using only proton data and $\gamma=1.5$. Top panel shows the entropy ratio against $r_{B}$, middle panel the magnetosonic Mach number against $r_{B}$, and bottom panel the Alfvén Mach number against $r_{B}$. Filled circles have entropy increases and open circles have entropy decreases.

better describe the plasma state in the regions local to shock waves. Values for $\gamma$ of 2 (Chao and Wiskerchen, 1974) or 3 (Papadopoulos, 1985) have been theoretically justified but the consequence of applying these to the data would be many more shock waves appearing to have entropy decreases.

An explanation for the calculated entropy decreases at some interplanetary shock waves could be envisaged if some aspect of the shock structure could be shown to be extended through space. In this case the free energy injected into the plasma at the shock wave boundary may not be completely thermalised immediately. This would result in lower than expected downstream temperatures and the effects should be visible in some aspect of the spacecraft observations. The magnetic field data, and to a lesser extent the plasma data, generally show a relatively sharp boundary between upstream and downstream states. However, a signature which often extends for hours into the downstream region can be seen in high frequency electrostatic field fluctuations. These plasma waves which are detected by the URAP instrument on Ulysses tend to be in the frequency range $1-50 \mathrm{kHz}$ and are a striking feature in daily dynamic spectra. 


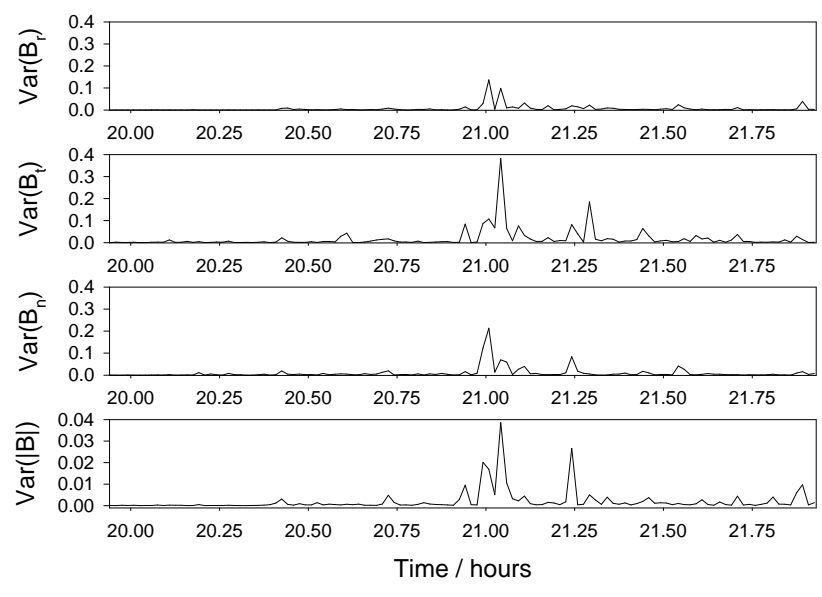

Fig. 7. Magnetic field variance in 3 components and magnitude (4 panels) using bins of $60 \mathrm{~s}$ of data for shock on day 326, 1996. The three components are in the standard heliospheric $\mathrm{r}, \mathrm{t}, \mathrm{n}$ coordinate system.
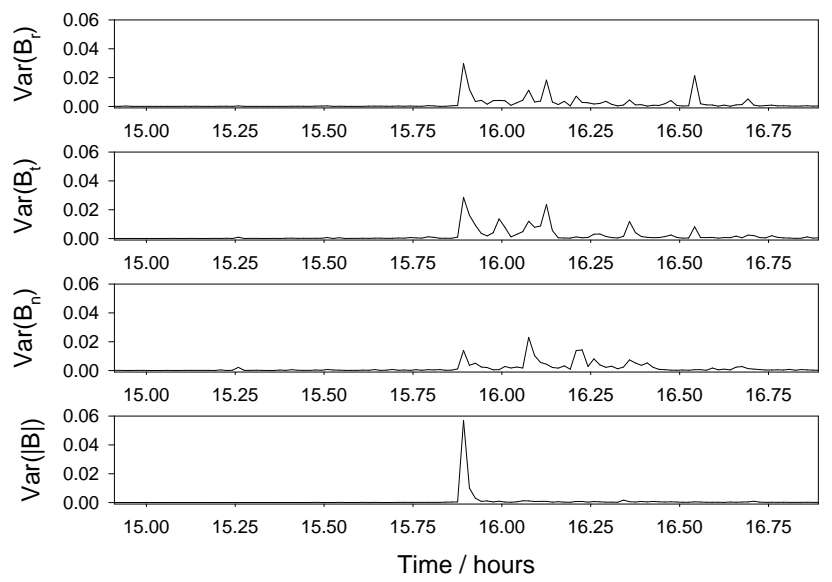

Fig. 8. Magnetic field variance in 3 components and magnitude (4 panels) using bins of $60 \mathrm{~s}$ of data for shock on day 296, 1997. The three components are in the standard heliospheric $r, t, n$ coordinate system.

In order to investigate the extent of shock structure in the magnetic field data, a variance analysis of several events has been performed. Data regions of $1 \mathrm{~h}$ are taken on either side of the shock waves and the variance of each component and magnitude of the magnetic field is calculated using consecutive 1-min periods of data. The results of this analysis are shown for a relatively strong quasi-parallel and quasiperpendicular shock in Figs. 7 and 8, respectively. In both cases, there is a strong increase in the variance of all 3 components and magnitude of the magnetic field at the shock transition itself. By comparing the scales of these two variance plots it is clear that the fluctuations in the three components of the quasi-parallel shock are considerably greater than in the magnitudes of either shock or the components of the quasi-perpendicular shock. The variance of the magnetic field remains at a higher level in the downstream region
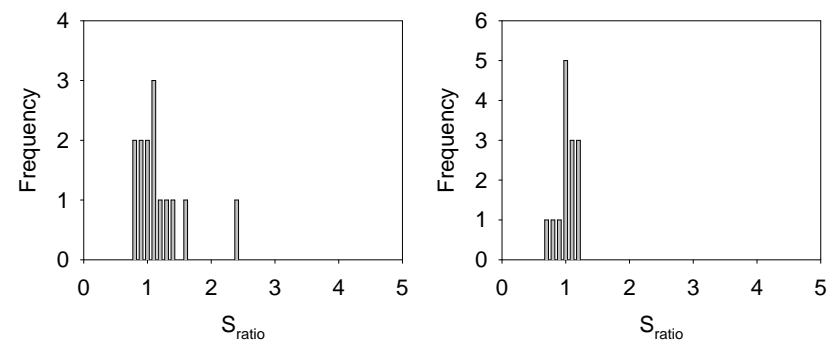

Fig. 9. Histograms of entropy fluctuations in 1-h up and downstream regions of 2 shock waves. Left panel: Shock with $r_{B}=2.54$ on day 326, 1996 showing the shock entropy transition ratio of 2.33 . Right panel: Shock with $r_{B}=1.56$ on day 246,1996 showing the shock entropy transition embedded in the background fluctuations.

in both cases although for the quasi-perpendicular shock the fluctuations are almost all in the three-magnetic field components, whereas for the quasi-parallel shock the fluctuations are in the magnetic field magnitude as well. In the case of the quasi-parallel shock there is also increased variance in a short region just upstream of the shock which is an expected feature of this magnetic field geometry. The analysis has also been performed for a quasi-parallel and quasi-perpendicular weak shock but in both cases the increase in variance is mainly confined to the shock transition itself. The magnetic variance analysis has shown that interplanetary shock waves can have an extended effect into a considerable downstream region but that this is a property of stronger shock waves. This does not provide an insight into the extent of thermalisation processes at the weaker shock waves.

All the shocks under consideration in this study are relatively weak events and some are extremely weak. Particularly in the weakest cases it is possible that the surrounding entropy fluctuations are significant. To demonstrate the relative importance of these fluctuations, entropy ratios have been calculated for consecutive sets of two plasma data points from $1 \mathrm{~h}$ upstream to $1 \mathrm{~h}$ downstream of two shock waves. The first event is a relatively strong shock and the second is a weaker shock. The results of these calculations are shown in Fig. 9 as histograms of entropy ratios calculated over two-hour periods. It is expected that the entropy change at the shock wave should be clearly distinguished and higher than the surrounding entropy fluctuations. In the lefthand panel of Fig. 9, which shows the results for the stronger shock, the entropy change at the shock transition can clearly be seen above the distribution of background fluctuations. However, in the right-hand panel, which shows the results for the weaker shock, the entropy change due to the shock transition is embedded within the distribution. This shows that in the case of the weaker shocks, the background entropy fluctuations are comparable to the entropy change at the shock transition. These results demonstrate the importance of using 10-min up and downstream periods for the calculation of entropy across the weaker shock waves, to ensure that the calculation is not drowned by background fluctuations. 
There are other important issues that can contribute to understanding the entropy results. The shock waves being analysed here are generally weak interplanetary shocks, some of which may be in the process of decaying and losing their shock structure. The processes occurring in these weaker shocks are not all that well understood and choosing up and downstream regions may be complicate by the fact that different processes happen over different times cales.

\section{Conclusions}

The thorough analysis of 116 interplanetary shock waves in terms of their shock parameters has produced some interesting and unexpected results. All the shock waves are found to have magnetic parameters which agree with the MHD shock theoretical predictions. However, for a three-component adiabatic plasma 61 shocks do not show the expected entropy increase across the shock and it is also found that 18 shocks have magnetosonic Mach numbers which are less than 1 . Three of these 18 shocks also have $r_{B}$ values less than 1 and these are 3 slow shock candidates. The use of longer data sets, $1 \mathrm{~h}$ up and downstream of the shocks, has not provided an answer to the entropy problem. This means that there is no clear entropy increase even if longer regions up and downstream are considered. The reason for this is that naturally occurring entropy fluctuations in the solar wind appear to mask any increase specifically caused by the weakest shock waves.

By using only proton data, radial temperatures and an empirical value of $\gamma=1.5$ the number of shocks failing the entropy condition is reduced to 14 . Clearly, an entropy decrease at any of these shocks waves would be a contradiction. The use of the empirical value of $\gamma=1.5$ appears, therefore, to be inappropriate, as it may well reflect (empirically) the largescale solar wind plasma but not the properties local to the shock waves.

The theoretically more acceptable values, $\gamma=\frac{5}{3}, 2$ or 3 , as discussed above, lead, as has been shown, to an even greater discrepancy. A value of $\gamma$ closer to 1 would lead to a non-decreasing entropy at most or all shocks, however, it would be difficult to justify such an arbitrary and small $(\sim 1)$ value for $\gamma$. The present analysis does not lead to a satisfactory explanation of the entropy measurements and more work is needed, but primarily, higher resolution plasma measurements in the vicinity of these weak interplanetary shocks are required in the future.

The extent of shock influence into up and downstream regions has been considered and both high frequency electrostatic waves and magnetic field fluctuations are found to persist for significant periods downstream of the events. However, the variance analysis also suggests that magnetic fluctuations are not so prominent in the case of weaker shocks. Some of the shock waves analysed in this study are events which lie close to the edge of what can be considered a shock wave and inevitably as this point is approached their structure becomes progressively submerged in the surrounding solar wind. This work has revealed a sensitivity to the data involved in some of the calculations which reflects both the weak state of some of the events and the difficulties in the determination of the temperature and further thermodynamic properties in these situations.

Acknowledgements. We are grateful to the SWOOPS experiment (Principal Investigator, D. J. McComas) on the Ulysses spacecraft for supplying the plasma data used in the shock wave analysis. We are also grateful to the UK Particle Physics and Astronomy Research Council that supports Ulysses related work at Imperial College.

Topical Editor T. Pulkkinen thanks a referee for his/her help in evaluating this paper.

\section{References}

Balogh, A., Beek, T. J., Forsyth, R. J., Hedgecok, P. C., Marquedant, R. J., Smith, E. J., Southwood, D. J., and Tsurutani, B. T.: The magnetic field investigation on the Ulysses mission: instrumentation and preliminary scientific results, Astron. Astrophys. Suppl. Ser., 92, 221-236, 1992.

Balogh A., González-Esparza, J. A., Forsyth, R. J., Burton, M. E., Goldstein, B. E., Smith, E. J., and Bame, S. J.: Interplanetary shock waves: Ulysses observations in and out of the ecliptic plane, Space Sci. Rev., 72, 171-180, 1995.

Bame S. J., McComas, D. J., Barraclough, B. L., Phillips, J. L., Sofaly, K. J., Chavez, J. C., Goldstein, B. E., and Sakurai, R. K.: The Ulysses mission solar wind plasma experiment, Astron. Astrophys. Suppl. Ser., 92, 237-265,1992.

Chao K. C.: Steepening of Nonlinear Waves in the Solar Wind, J. Geophys. Res., 25, 5411-5424, 1973.

Chao, J. K. and Wiskerchen, M. J.: The ratio of specific heats for postshock plasmas of a detached bow shock: An MHD model, J. Geophys. Res., 79, 31, 4769-4774, 1974.

Chao, J. K. and Hsieh, K. C.: On determining Magnetohydrodynamic Shock Parameters $\theta_{B n}$ and $M_{A}$, Planet. Space Sci., 32, 641-646, 1984.

Colburn, D. S. and Sonett, C. P.: Discontinuities in the solar wind, Space Sci. Rev., 5, 439-506, 1966.

de Hoffmann, F. and Teller, E.: Magneto-Hydrodynamic Shocks, Phys. Rev., 80, 692-703, 1950.

Gonzáles-Esparza J. A.: Study of heliospheric shock waves observed by Ulysses Magnetometer in and out of the ecliptic plane, PhD thesis, Imperial College, 1995.

Gonzáles-Esparza J. A. and Balogh, A.: The $\theta_{B n}$ problem: Determination of local magnetic parameters of interplanetary shocks from in situ IMF data, Geophísica Internacional, 40, 1, 53-63, 2001.

Gosling, J. T., Pizzo, V., and Bame, S. J.: Anomalously low proton temperature in the solar wind following interplanetary shock waves: Evidence for magnetic bottles?, J. Geophys. Res., 78, 2001-2009, 1973.

Hsieh, K. C. and Richter, A. K.: The Importance of Being Earnest About Shock Fitting, J. Geophys. Res., 91, 4157-4162, 1986.

Liu, S., Marsch, E., Livi, S., Woch, J., Wilken, B., von Steiger R., and Gloeckler, G.: Radial gradients of ion densities and temperatures derived from SWICS/Ulysses observations, Geophys. Res. Lett., 22, 18, 2445-2448, 1995. 
Liu, S., Marsch, E., Schwenn, R., and Rosenbauer, H.: A method for shock determination and classification., American Institute of Physics, 1996.

Papadopoulos, K., Stone, R. G., and Tsurutani, B. T.: Microinstabilities and anomalous transport Geophysical Monograph, 34, 1985.

Richter, A. K., Rosenbauer, H., Neubauer, F. M., and Ptitsyna, N. G.: Solar Wind Observations Associated With a Slow Forward Shock Wave at 0.31 AU, J. Geophys. Res., 90, 7581-7586, 1985.
Smith, E. J. and Burton, M. E.: Shock Analysis: Three Useful New Relations, J. Geophys. Res., 93, 2730-2734, 1988.

Sonett, C. P., Colburn, D. S., Davis, L., Jr., Smith, E. J., and Coleman, P. J., Jr.: Evidence for a collision-free magnetohydrodynamic shock in interplanetary space, Phys. Rev. Lett., 13, 153156, 1964.

Totten, T. L., Freeman, J. W., and Arya, S.: An empirical determination of the polytropic index for the free-streaming solar wind using Helios 1 data, J. Geophys. Res., 100, 13-17, 1995. 